\title{
Channel Photomultiplier electronic module enables light measurement over 9 decades including single photon detection.
}

\author{
Steigel, Dirk; Jesch, Hartmut; Reichert, Willi; Gauer, Mario; Barden, Raimund \\ PerkinElmer Optoelectronics GmbH \& Co. KG \\ Wenzel-Jaksch-Straße 31, 65199 Wiesbaden, Germany
}

\begin{abstract}
Optical detector modules based on Channel Photomultiplier technology have been developed with a dynamic range of 9 decades $\left(10^{9}\right)$. Sensitivity ranges from the detection of single photons up to a count rate equivalent of $1 \mathrm{Gcps}$ (Giga counts per second). Applications for the new CPM module include high throughput screening, time resolved fluorescence, luminescence and real-time PCR.
\end{abstract}

\section{Introduction}

Applications in analytical and life science instrumentation e.g. for fluorescence and luminescence measurements, require optical detectors with single photon sensitivity and very high dynamic range. The typical detector for such applications is a photomultiplier tube (PMT). Over the last years also semiconductor types of photomultipliers have been introduced, though often with a high background noise, even when strongly cooled. Channel Photomultiplier (CPM) detector technology, introduced some years ago, has a proven very low background and high linearity over a wide dynamic range. In this paper, we present a new electronic module designed to exploit these advantages to the full in CPM's and other similar devices.

The classical approach to detect single photons is the so-called photon counting technology. This technology uses the amplified electronic pulses of the photomultiplier or a similar device created by a photon and counts all anode pulses which are higher than a certain pulse height. Even single photons can be detected accurately, despite any background noise. However at high count rates, the amplified anode pulses start to overlap and single pulses can no longer be distinguished. Traditional photon counting technology is therefore limited to moderate count rates and yields only restricted linearity. Depending on a photomultiplier's pulse width response, linearity is limited to typically some tens of Mcps (Mega counts per second). Therefore photon counting units can normally cover only approx. up to seven orders of magnitude dynamic range.

At higher photon intensities, the conventional photo-current detection is used to measure the light intensity, the so-called DC mode of operation in a PMT or CPM, but this mode typically has not been used to detect single photons. There have been approaches to extend the dynamic range by combing photon counting mode with DC mode; however there is always the difficulty how to fit both linear curves measured in different detection modes.

In this paper we report the development of a new readout technique, which enables ultra-low-light measurements in DC operation mode but including single photon detection. In contrast to the traditional

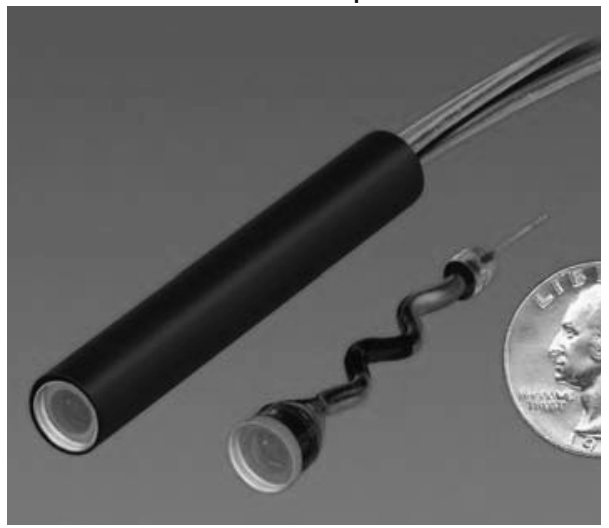
photon counting operation mode the new readout technique overcomes the performance limitations at high light levels. The new readout technique takes advantage of the unique feature of the CPM namely the low background characteristics. As a result, measurements can be performed from single photon level with minimal background up to high light level over 9 decades. Moreover this technique can be applied to other detector types to enhance their dynamic range.

Fig.1: Photograph of the Channel Photomultiplier. Left shows the encapsulated CPM; at the right the bare CPM without encapsulation is shown. 


\section{Principle of the Channel Photomultiplier}

Fig. 1 shows a photograph and Fig. 2 a schematic of the new Channel Photomultiplier (CPM): Like in the case of a conventional head-on photomultiplier tube (PMT) light illuminates a semi-conductive photocathode which is deposited on an entrance window. Inside the photocathode light is converted into photoelectrons due to the photoelectrical effect. Photoelectrons are emitted into the vacuum and are accelerated in direction to the anode via a high electrical field. On the way to the anode the electrons have to pass through a thin, bent, semi-conductive channel. Each time when electrons hit the wall of the channel, several secondary electrons are emitted from the surface. Again these secondary electrons are accelerated down the channel, hit the channel surface and create still more secondary electrons. This process repeats multiple times. Ultimately, an avalanche effect with an electron multiplication factor exceeding 100 million can be obtained. The resulting current is read out at the anode. The curved shape of the channel provides a low-noise multiplication effect and prevents possible ion feedback. The electron tube is encapsulated inside a plastic housing and held with a silicone rubber potting material.

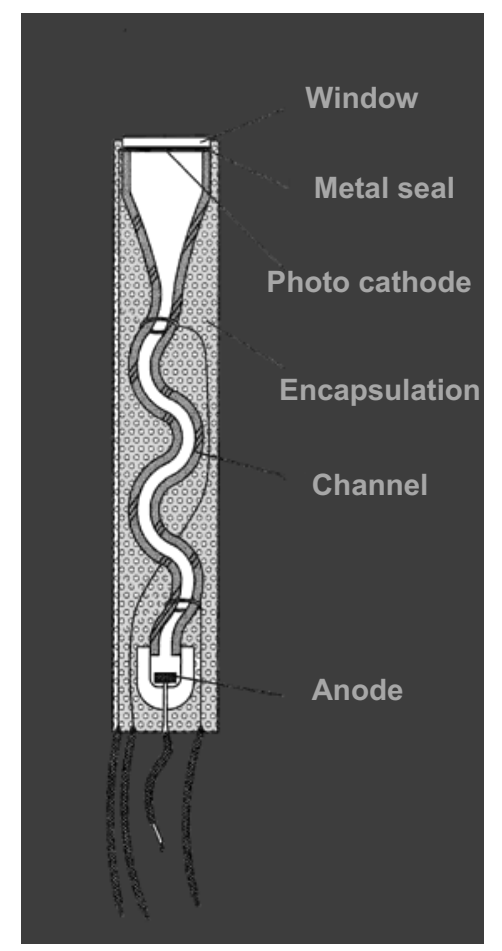

Fig.2: Schematic view of a channel photomultiplier. Photons are converted into photo electrons in the photocathode and are accelerated towards the anode. On the way to the anode the electrons pass a hallow channel. At each collision the number of electrons is multiplied. At the anode a gain of up to 100 millions can be reached.

\section{CPM background characteristics}

A significant advantage of the CPM technology is the very low background. The term background (or anode dark current) refers to the measured output signal in the absence of photocathode illumination. For a low noise Bialkali CPM the anode dark current is only approx. $1 \mathrm{pA}$ at a gain of $1 \times 10^{6}$. Typical anode dark current values of conventional photomultipliers are characterized in low nA-region. Typically the anode dark current level of the CPM is one to three orders of magnitude lower in respect to conventional photomultiplier tube (PMT).

Because of the low background level of the CPMs, an extended dynamic range is achieved in comparison to conventional PMT.

The main reason for the low background is the fact that electron multiplication in the channel is virtually noise-free. Measurements of background in channel electron multiplier show a dark count rate of 0.01 to 0.1 counts per second. With conventional photomultipliers the background originating from the electron multiplier (dynode structure) is generally a non-negligible part of the total background.

Another reason for the low background is the negligible leakage current in the anode circuit. The anode is electrically highly insulated because during photocathode activation, alkali contamination of the tube bodies including anode parts is prevented. The high voltage potential of the cathode is spatially well separated from the anode. The signal readout is enabled using low-noise $50 \mathrm{Ohm}$ Coax cable.

As a result of the virtually noise free channel and negligible leakage currents in the anode circuit, the photocathode is in practice the sole contributor to the CPM background. The thermionic emission current, being the reason for the background, varies for the different types of photocathode materials: For solely UV sensitive photo cathodes thermionic background is negligible. For the visible spectral range special low background photo cathodes have been developed: The low noise Bialkali for the blue and green range and the Yellow extended for the range up to $700 \mathrm{~nm}$. Multialkali types of photo cathodes take the spectral range up to $900 \mathrm{~nm}$. Fig. 3 shows the spectral sensitivity of different photo cathode materials. 


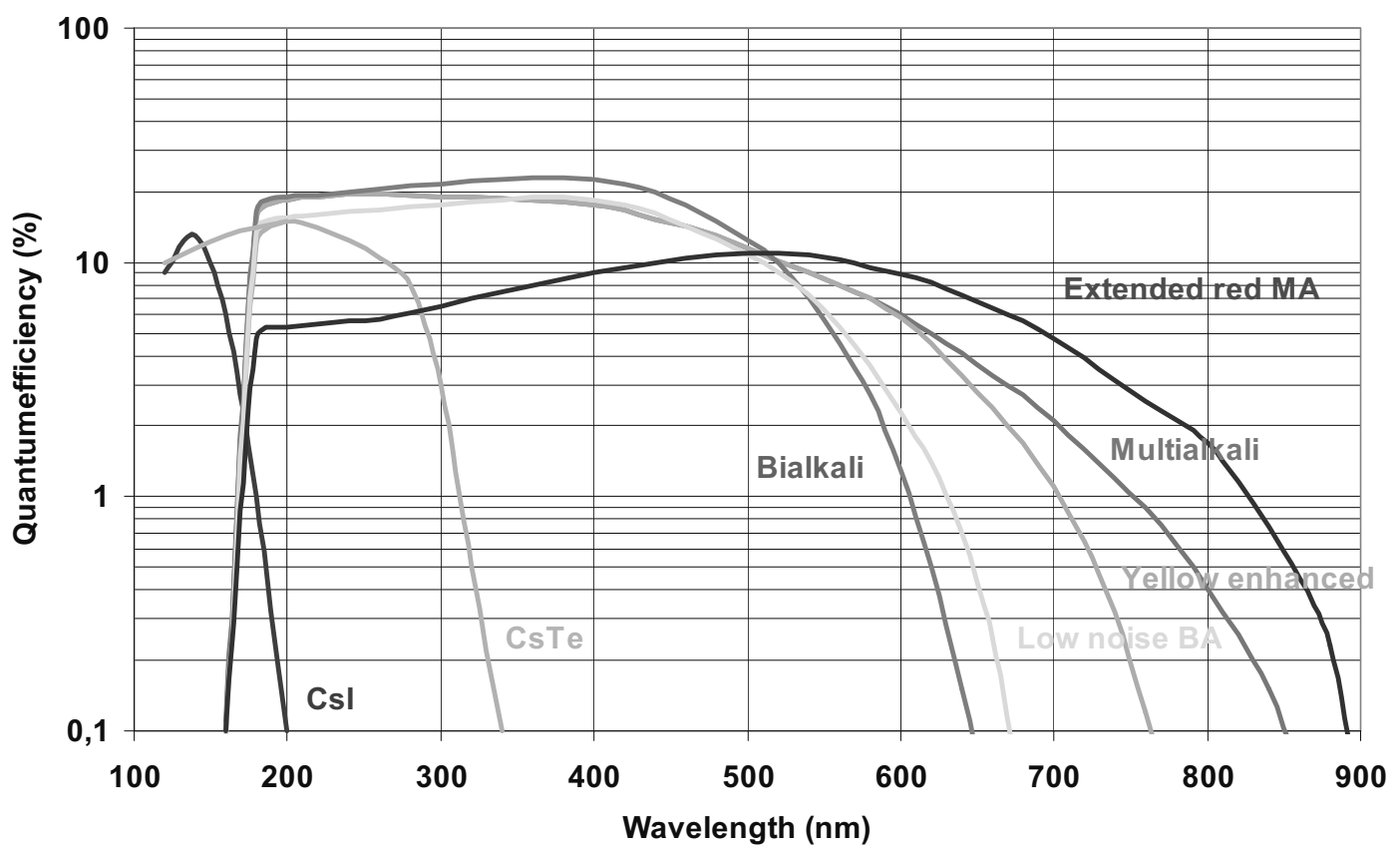

Fig.3: The quantum efficiency vs. wavelength is given for a variety of photo cathodes: The solar blind types $\mathrm{CsI}$ and CsTe cover the UV range. Photo cathodes for the visible range are the Bialkali, the low noise Bialkali and the Yellow enhanced. The visible to near infrared range is covered by the Multialkali and extended red Multialkali.

\section{CPM detection limits for ultra low light levels}

Due to the extremely low dark current the ability to detect very weak light signals is superior for the CPM in respect to classical PMTs. The smallest detectable light limits can be expressed by the equivalent noise input (ENI) or noise equivalent power (NEP). The ENI refers to the amount of light which is necessary to produce a signal-to-noise ratio of unity. The ENI values are expressed in watts for a $1 \mathrm{~Hz}$ detection bandwidth. Usually dynode based PMTs have values in the $10^{-15}$ to $10^{-16} \mathrm{~W}$ range. Fig. 4 shows the situation for the different types of CPMs. ENI values down to the $10^{-18} \mathrm{~W}$ range corresponding to a photon flux of approx. 10 photons per second can be achieved with Channel Photomultipliers.

The ENI values demonstrate the advantage of the CPM technology. For the detection of extremely low amounts of light which is especially important in applications like fluorescence analysis and bio or chemo luminescence the lowest ENI values guarantees lowest detectable limits. The photocathode types low noise Bialkali for the blue / green spectral range) and yellow enhanced (for green / yellow range) are therefore the best suitable in such applications.

\section{Novel electronic readout}

Although CPM detectors are able to detect single photons and can also detect photon fluxes over many decades of dynamic range there has been a lack of electronic readouts to cover effectively the achievable entire dynamic range of the CPM.

The presented technical approach applies an electronic readout by using the measurement of the anode DC current and not the classical photo counting technique. Fig.5 shows the schematics of the electronic readout. The CPM anode current is readout by the input stage via ADC into a microcontroller. The microcontroller is readout via a USB interface to the PC. Also high voltage setting can be controlled by microcontroller to enable CPM gain to be switched. 


\section{Equivalent Noise Input}

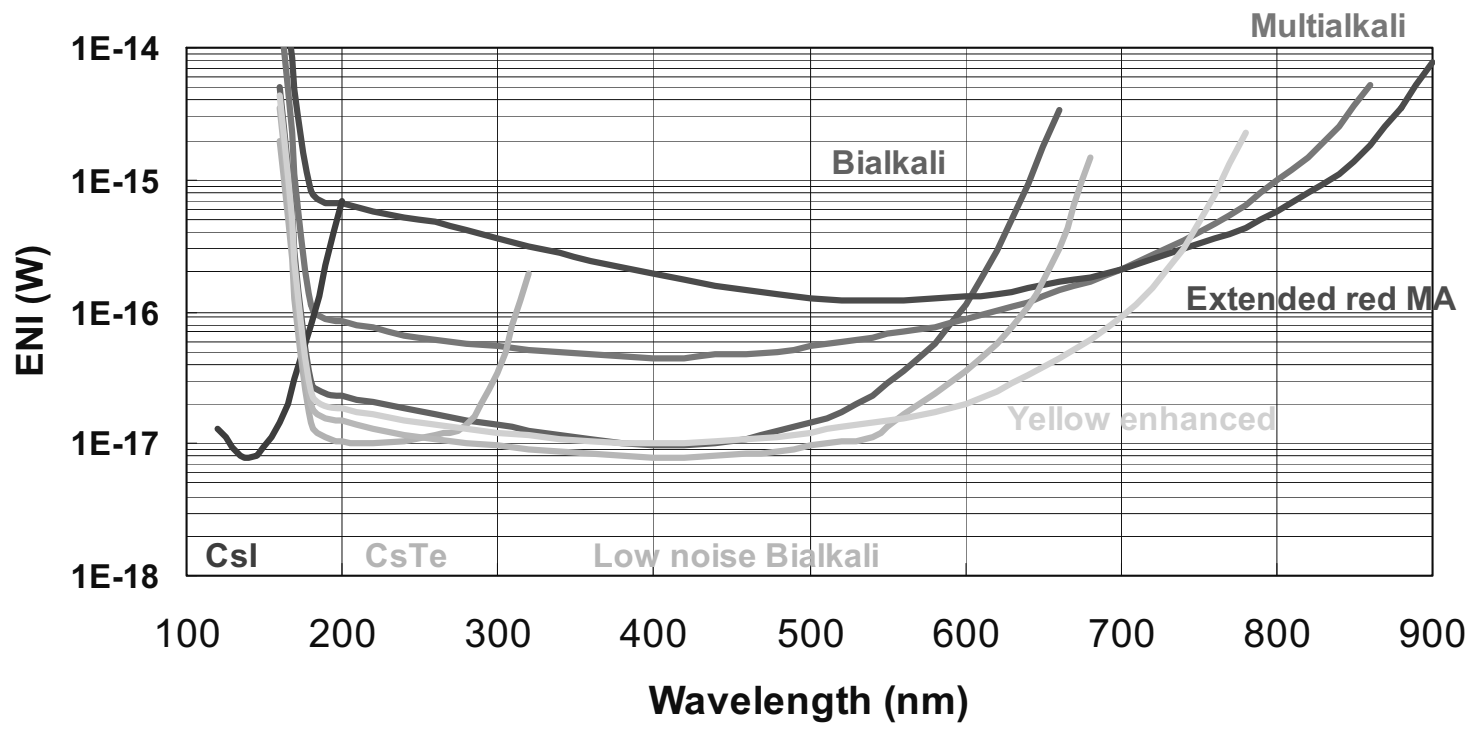

Fig.4: Equivalent noise input is shown vs. wavelength for different photocathodes of $1 / 3$ " CPM. The equivalent noise input is a value for the lowest detectable amount of light energy. For several photocathode types light energies in the range of $10^{-17} \mathrm{~W}$ or even lower can be detected.

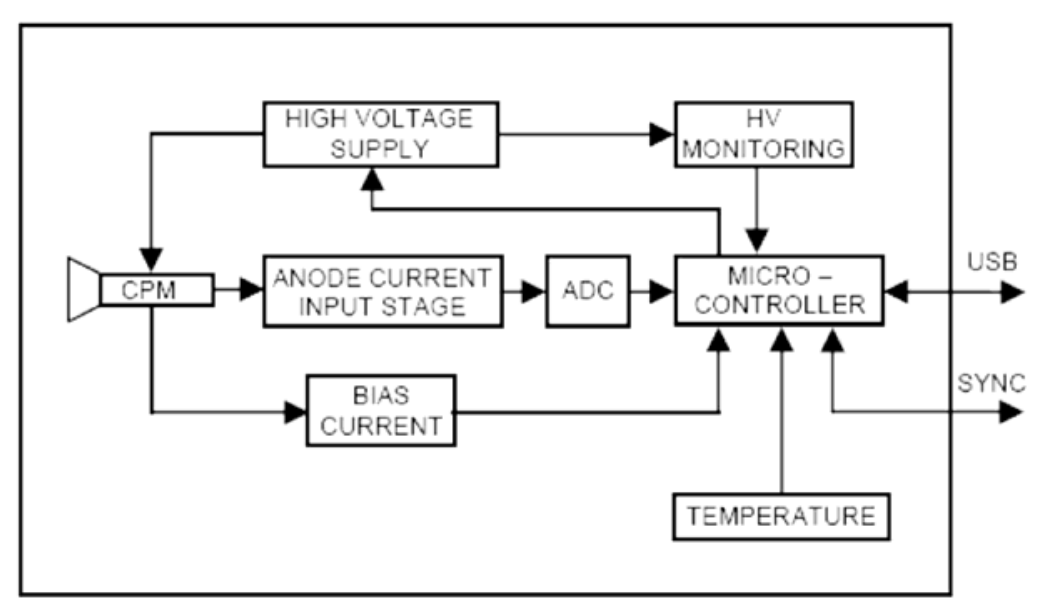

Fig.5: Schematics of the readout electronics

Here the special feature of the CPM that the background is impacted only by thermionic current of the photocathode is used. This feature allows to determine DC signals without any additional other noise from the background. As result and proven by experiments, background measured in DC analogue mode corresponds to the dark counts measured in photon counting mode.

\section{Linearity measurements}

Linearity measurements with the new readout electronics show linear characteristics up to photon fluxes of approx. $2 \times 10^{8}$ photons per second (equivalent to a count rate of about 100 Mcps with $7-8$ decades of linearity). Up to two orders of magnitude higher photon fluxes $\left(5 \times 10^{10}\right.$ photons per second) can be measured by a high voltage CPM gain switching. Fig. 6 shows linearity behavior at different high voltage setups.

To increase the linearity further the new readout electronics also provides a readout mode with extended dynamic range. In this mode the linearity is extended to a count rate equivalent of approx. $1 \mathrm{Gcps}$ (Fig.7). The extension of the linearity is provided by fast gain switching. Preliminary results indicate that even count rates equivalents exceeding $10 \mathrm{Gcps}$ are feasible using this technique. 


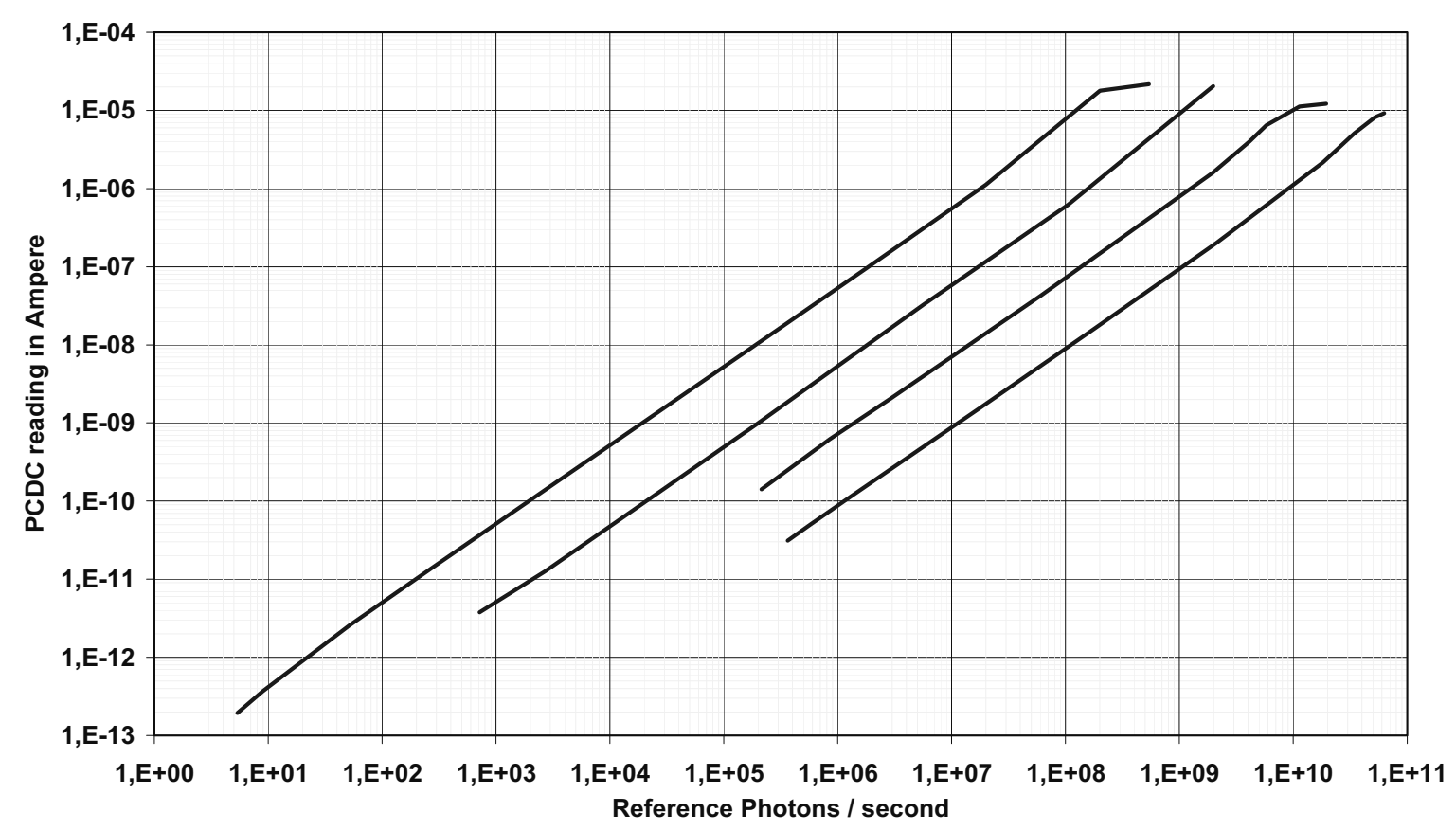

Fig.6: Linearity measurements show linear characteristics from a photon flux of a few counts per second up to $10^{8}$ photons per second range (upper curve). Photon fluxes from a few photons per second up to the $10^{10}$ range can be measured by high voltage switching (see the lower curves).

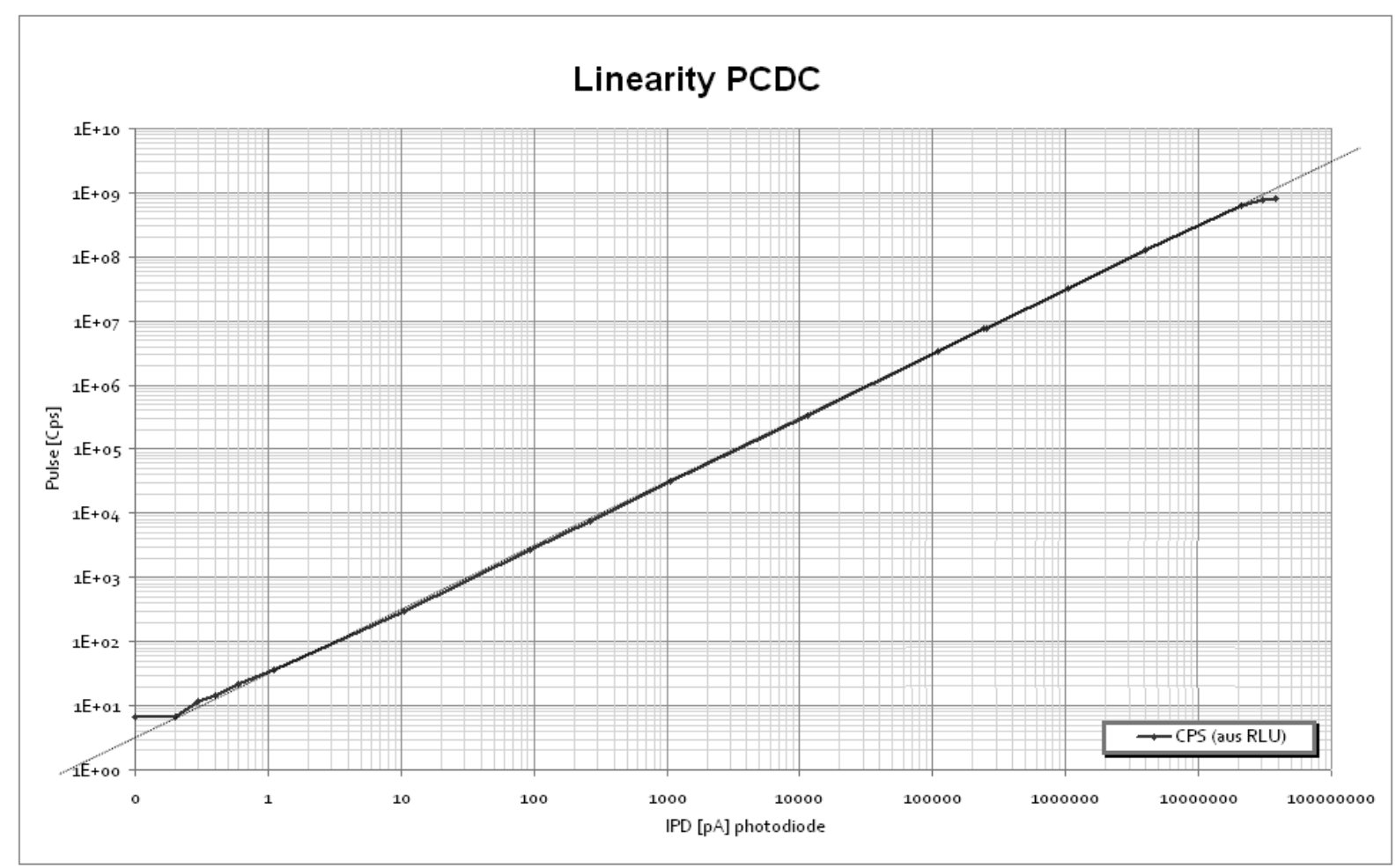

Fig.7: Linearity measurements using the extended dynamic range show to a maximum count rate equivalent of approx. $1 \mathrm{Gcps}$. 


\section{PCDC modules}

Since the new electronic readout combines the advantages of Photon counting and analogue DC mode it is called "PCDC". The electronics is incorporated in an electronic module including CPM, high voltage power supply and USB interface. Module is shown in Fig. 8.

Additional features like the synchronization I/O offer the possibility to synchronize the measurement with other devices in the application like flash lamp trigger or laser or LED pulse for example. The incorporated electronic shutter function can be applied to protect the detector against over-illumination with high light level excitation from excitation lamp or laser.

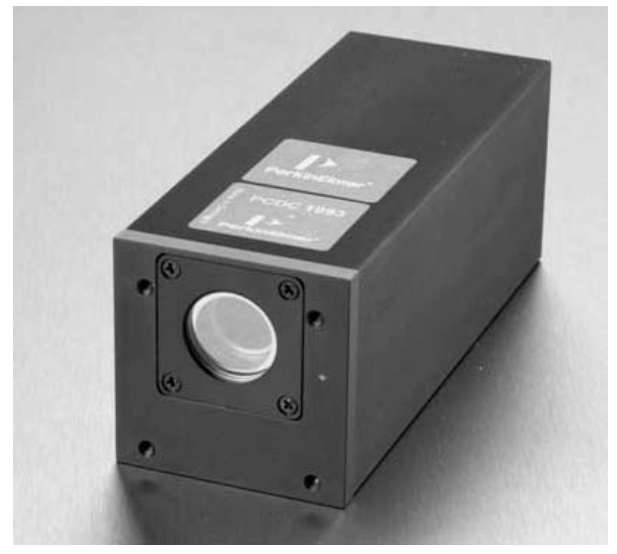

Fig.8: PCDC Module including CPM, high voltage power supply and readout electronics with USB interface

\section{Applications for the PCDC modules}

The PCDC modules combine the advantages of lowest detectable limits for ultra weak light levels of the CPM technology with the high dynamic range of the novel electronic readout. These features allow earlier, more secure and faster detection of indicators for specific diseases in medical diagnostics. It enables faster screening in the research and development for new pharmaceuticals in medical therapy and enables higher detectable limits for the detection and monitoring traces and impurities in environmental and chemical analysis. In the following some examples of applications are given, where the performance of the PCDC module is advantageous:

\section{a. Bio- and chemo-luminescence}

Bio and chemo luminescence application in general have very low background from the probes so the low background characteristics of the CPM is advantageous. Usually those measurements are made in photon counting mode. With the PCDC module the same background performance can be obtained but we gain dynamic range by more than one order of magnitude.

\section{b. Time resolved fluorescence}

Time resolved fluorescence measurements can be made by using different high voltage settings as shown in Fig. 6. Due to the high linearity of the PCDC, luminescence and fluorescence mode can be combined with one PCDC module. Possible over-illumination by excitation lamp or laser can be avoided by the usage of the incorporated electronic shutter.

\section{c. High through-put screening}

High through-put screening of micro-titre plates needs to be very fast. Due to the high dynamic range of the PCDC, the usage of time consuming filter wheels settings with different ND filters can be avoided.

\section{d. DNA analysis with real-time PCR}

To monitor the multiplication of DNA in real time PCR new detection systems are requested with highest dynamic range. PCDC covers the 9 orders of magnitude dynamic range and handles completely the light detection management inside the instrument from a photon flux of a few photons per second up to levels in the $10^{9}$ photons per second and higher.

\section{Conclusion}

We have reported a new electronic amplifier module for use with Channel Photomultiplier CPM and similar detectors, allowing single photon detection at lowest background and a linear response over 9 decades of light intensity. This module will find numerous applications in analytical instrumentation for human and environmental health. 